\section{Demencia reversible por neurosífilis (parálisis general progresiva)}

\section{Sr. Director:}

La neurosífilis es la afectación del sistema nervioso central por el Treponema pallidum. La parálisis general progresiva (PGP) constituye uno de los síndromes tardíos de la neurosífilis, siendo en la era pre-antibiótica una de las causas más frecuentes de ingreso hospitalario por demencia. En el año 2003, tras la estabilización del número de los casos notificados en los años anteriores, se ha advertido un incremento en la incidencia de sífilis, situándose en una tasa de 2,19/100.000 habitantes (1). Resulta difícil obtener datos fiables sobre la incidencia real de neurosífilis pero se estima que puede presentarse en un 4-10\% de los afectados de sífilis (2).

Se trata de un varón de 46 años, fumador ,consumidor de 40 gr. alcohol/día, sin otros factores de riesgo cardiovascular y con contactos de riesgo para enfermedad de transmisión sexual (ETS). No refería antecedentes de traumatismo craneoencefálico (TCE) reciente ni toma de fármacos y nunca había sido diagnosticado ni tratado de lúes.

El paciente es atendido inicialmente en los Servicios de Salud Mental por sospecha de trastorno depresivo con pseudodemencia, supuestamente reactivo a situación de ruina económica sufrida recientemente. Después de ser tratado con sertralina y cloracepato dipotásico, sin obtener mejoría durante mes y medio, se deriva al paciente al hospital, para descartar proceso neurológico orgánico.

$\mathrm{Al}$ ingreso el paciente estaba consciente, con un cuadro fluctuante de deterioro de la memoria reciente, alteraciones en el curso del pensamiento, desorientación temporal, acalculia y alteraciones del comportamiento con conductas extrañas. En la exploración neurológica presentaba pupilas de Argyll-Robertson (pupila pequeña e irregular que acomodaba para la visión cercana pero no ante estímulos lumínicos). El resto de pares craneales, asî como la fuerza y la sensibilidad, eran normales. Los reflejos osteotendinosos estaban exaltados y en las pruebas cerebelosas no se encontraron alteraciones. Los reflejos de liberación frontal fueron positivos (grasping, palmo-mentoniano, hociqueo-chupeteo y perseverancia motora).

Entre las pruebas complementarias solicitadas el hemograma, la coagulación, la bioquímica básica, el espectro electroforético sérico y los niveles de vitamina B12, ácido fólico y TSH resultaron todos dentro de límites normales. La serología VIH y los anticuerpos antinucleares fueron negativos. La radiografía de tórax, la TAC craneal y el electroencefalograma resultaron normales. Se realizó una punción lumbar obteniéndose un líquido cefalorraquídeo de aspecto cristalino con presión de apertura en límites normales $\left(110 \mathrm{~mm} \mathrm{H}_{2} \mathrm{O}\right)$; leucocitos $40 / \mu \mathrm{l}$ (96\% mononucleares); glucosa $54 \mathrm{mg} / \mathrm{dl}$; proteínas $80 \mathrm{mg} / \mathrm{dl}$; tincion de Gram negativo y cultivo estéril; auramina y Rosa de Bengala negativas; VDRL (veneral disease reaserch laboratory) positivo con hemaglutinación positiva.

Con el diagnóstico de neurolúes (parálisis general progresiva), se procede al tratamiento con penicilina G acuosa IV a dosis de $24 \times 10^{6}$ unidades durante 14 días, tras el cual el paciente experimentó una progresiva mejoría del comportamiento y capacidades intelectuales. En los controles posteriores de LCR la serología, proteínas y células fueron normalizándose progresivamente.

La neurosífilis es la afectación del SNC por Treponema pallidum. La PGP es uno de los síndromes clínicos tardíos de la neurosífilis que puede presentarse entre 5 y 40 años después de la primoinfección (Tabla I). En la época pre-antibiótica era una de las causas más frecuentes de ingreso hospitalario por demencia. En nuestros días, ha pasado a ser una forma de presentación infrecuente, hasta la aparición del SIDA (3).

Las manifestaciones de la PGP indican la existencia de lesiones parenquimatosas extensas. Inicialmente, suelen predominar los síntomas psiquiátricos, con dificultad de concentración, pér-

TABLA I

CLASIFICACIÓN CLINICOPATOLÓGICA DE LA NEUROSÍFILIS

Neurosífilis asintomática (latente)

Neurosífilis meningea, meningovascular y goma:

-Precoz

-Tardía

Neurosífilis vascular

Neurosífilis parética ( paralisis general progresiva, demencia paralítica)

Neurosífilis tabética

Ocular ( atrofia y neuritis óptica aguda)

Lesión significativa del VIII par

Meningoencefalitis sifilítica ( amiotrofia sifilítica, paraplejía espástica de Erb) 
dida de memoria reciente e ideas delirantes. Posteriormente, se instaura una demencia franca con desorientación y trastornos del juicio, cálculo y razonamiento, así como reflejos osteotendinosos exaltados y pupilas de Argyll-Robertson .

El diagnóstico de neurosífilis se basa en las características clínicas referidas anteriormente, junto con las anomalías biológicas y serológicas de LCR.

El examen de LCR es esencial para evaluar a cualquier paciente con serología de lúes positiva en sangre que presenta signos y síntomas neurológicos. Se aconseja también analizarlo en todos los pacientes con sífilis no tratada de duración desconocida, en aquellos pacientes con una evolución de más de un año o si hay complicaciones tardías diferentes de la neurosífilis.

El recuento de células en LCR muestra una pleocitosis linfocitaria $\left(15-500 \mathrm{cél} / \mathrm{mm}^{3}\right)$ y las proteínas experimentan un incremento moderado (45-200 $\mathrm{mg} / \mathrm{dl})$.

El test de elección para el diagnóstico de neurosífilis es el VDRL en LCR (especificidad del 100\%) (4), aunque su sensibilidad es muy baja (40-60\%). El FTA- Abs es mucho más sensible que el VDRL pero pueden darse falsos positivos con mucha frecuencia debido al paso de anticuerpos a través de la barrera hematoencefálica o punciones lumbares traumáticas (5). Con el fin de evitar los falsos positivos, se han diseñado índices de pruebas treponémicas que estiman la producción intratecal de anticuerpos, siendo el más sencillo el cociente THPA (Treponema pallidum hemagglutination test) que se obtiene de la siguiente manera:

Indice THPA: título THPA en LCR/Cociente albúmina

Cociente de albúmina = concentración albúmina LCR/concentración albúmina sangre.

Un resultado entre 70 y 500 indica probable neurosífilis activa y un índice $>500$ es prácticamente diagnóstico. La especificidad de este índice es alta, pero su sensibilidad no lo es tanto.

El diagnóstico diferencial se debe realizar con la patología susceptible de originar demencia (Tabla II) y pseudodemencia en cuadros depresivos importantes. En nuestro caso la asociación de alteraciones cognitivas y pupilas de Argyll-Robertson, prácticamente patognomónicas de neurosífilis parenquimatosa, orientó el diagnóstico hacia una PGP

Con el fin de detectar fracasos terapéuticos, relativamente frecuentes en la neurosífilis, se recomienda el estudio del LCR en los 3, 6, 12 y 24 meses después del tratamiento. La celularidad debe descender a los 3 meses, desapareciendo a los 6 meses. Las proteínas, sin embargo, descienden hasta normalizarse antes del año. Los títulos de VDRL deben desparecer o descender cuatro veces su valor, aunque es posible que queden positivos a títulos bajos $(<1 / 4)$ durante muchos años $(6)$. Si durante el seguimiento no se observa esta evolución en LCR o empeora la clínica se procede de nuevo al tratamiento con la misma pauta descrita anteriormente durante 14 días.

Ante todo lo expuesto, debido al aumento de la incidencia de sífilis en los últimos años y dada la potencial reversibilidad de las manifestaciones neuropsiquiatricas conviene tener en cuenta la neurolúes en el diagnóstico diferencial de pacientes con demencia.

\section{J. Galipienzo García, F. J. Imaz Pérez, J. García de Tena, A. García-Manzanares Vázquez, B. Zarza Sanz}

Servicio de Urgencias. Hospital Universitario Príncipe de Asturias. Alcalá de Henares. Madrid

1. Infecciones de transmisión sexual. Marzo 2005. Área de vigilancia epidemiológica. Centro Nacional de Epidemiología. Instituto de Salud Carlos III. Vigilancia epidemiológica del SIDA y el VIH en España. Estudios específicos.

2. Solbrig MV, Healy JF, Jay CA. Infections of the nervous system: Bacterial infections. I Bradley WG, Daroff RB, Fenichel GM, Marsden CD, eds. Neurology in clinical practice. Boston: Butterworth Heinemann 1999; p. 1317-51.

3. Sobhan T, Rowe HM, Ryan WG, Muñoz C. Unusual case reprt: Three cases of phychiatric manifestations of neurosyphilis. Psychiatr Serv 2004; 55 (7): 830-2.

4. Lunger AF, Schmidt BL, Kaulich M. Significance of laboratory findings for the diagnosis of neurosyphilis. International Journal of STD and AIDS 2000; 11: 224-34.

5. Lukehart SA, Hook, EW III, Baker-Zander, SA, Collier AC, Crichlow CW Handsfiel HH. Invasion of the central nervous system by Treponema pallidum: Implications for diagnosis and treatment. Ann Intern Med 1988; 109 ; 855-62.

6. Larsen SA, Steiner BM, Rudolph AH. Laboratory diagnosis and interpretation of test for syphilis. Clin Microbiol Rev 1995; 8; 1-21.

TABLA II

\begin{tabular}{|c|c|c|c|}
\hline \multicolumn{4}{|c|}{ CLASIFICACIÓN DE LAS DEMENCIAS } \\
\hline $\begin{array}{l}\text { Demencias primarias } \\
\text { Cortical }\end{array}$ & Subcortical & $\begin{array}{l}\text { Demencias secundarias } \\
\text { Vasculares }\end{array}$ & No vascular \\
\hline $\begin{array}{l}\text { Enfermedad de } \\
\text { Alzheimer }\end{array}$ & Enfermedad de Huntington & Demencia multiinfarto (demencia cortical) & $\begin{array}{l}\text { Origen infeccioso (encefalitis, SIDA, } \\
\text { neurosífilis, etc.) }\end{array}$ \\
\hline $\begin{array}{l}\text { Demencias fronto- } \\
\text { temporales (complejo } \\
\text { Pick) }\end{array}$ & $\begin{array}{l}\text { Parálisis supranuclear progresiva } \\
\text { Parkinson-demencia }\end{array}$ & $\begin{array}{l}\text { Demencia por enfermedad de pequeño vaso } \\
\text { (demencia subcortical) }\end{array}$ & $\begin{array}{l}\text { Origen metabólico, endocrino o } \\
\text { carencial (hipotiroidismo, enfermedad } \\
\text { Wilson, hipoxia-hipercapnia, fármacos, } \\
\text { alcohol, etc.) }\end{array}$ \\
\hline $\begin{array}{l}\text { Demencia con cuerpos } \\
\text { de Lewy }\end{array}$ & $\begin{array}{l}\text { Enfermedad de Creutzfeldt-Jakob } \\
\text { Otras formas (degeneración cortico- } \\
\text { basal, demencia mesolimbocortical, etc.) }\end{array}$ & $\begin{array}{l}\text { Demencia hemodinámica (isquemia-hipoxia): } \\
\text {-Infartos de zona frontera } \\
\text {-Infartos incompletos de sustancia blanca } \\
\text { Demencia hemorrágica: } \\
\text {-Hemorragia subdural } \\
\text {-Hemorragia subaracnoidea } \\
\text {-Hematoma cerebral } \\
\text { Demencia por infarto estratégico (demencia } \\
\text { talámica) } \\
\text { Otras formas (vasculitis, S. de Sneddon) }\end{array}$ & $\begin{array}{l}\text { Traumatismos craneales (demencia } \\
\text { postraumática, hematoma subdural } \\
\text { crónico) } \\
\text { Procesos expansivos (tumores o hidro- } \\
\text { cefalia a presión normal) } \\
\text { Otras (enfermedades desmielinizantes, } \\
\text { enfermedad de Labora, etc.) }\end{array}$ \\
\hline
\end{tabular}

